\title{
DIOPHANTINE EQUATIONS AND IDENTITIES
}

\section{MALVINA BAICA}

\section{Department of Mathematics and Computer Science University of Wisconsin - Whitewater Whitewater, Wisconsin 53190 U.S.A.}

(Received April 28, 1985)

ABSTRACT. The general diophantine equations of the second and third degree are far from being totally solved. The equations considered in this paper are

i) $x^{2}-m y^{2}= \pm 1$

ii) $x^{3}+m y^{3}+m^{2} z^{3}-3 m x y z=1$

iii) Some fifth degree diophantine equations

Infinitely many solutions of each of these equations will be stated explicitly, using the results from the ACF discussed before.

It is known that the solutions of Pell's equation are well exploited. We include it here because we shall use a common method to solve these three above mentioned equations and the method becomes very simple in Pell's equations case.

Some new third and fifth degree combinatorial identities are derived from units in algebraic number fields.

KEY WORDS AND PHRASES. Diophantine equations, identities, an algorithm in a complex field (abbr. $A C F$ ), units in the algebraic number fields. 1980 MATHEMATICS SUBJECT CLASSIFICATION CODE. 1OB10, 10B15, 10A30, $12 \mathrm{~A} 45$.

\section{O. INTRODUCTION}

In this paper we shall investigate Diophantine equations of the second and third degree of a special type. The general equations of the second and third degrees are far from being totally solved. It suffices to look up Mordell's book on Diophantine equations, to learn how little we actually know about the general second and third degree Diophantine equations, in spite of the many numerous results on this subject that have been gained by great mathematicians with no little effort. The famous Thue theorem stating that the equation

$$
\begin{aligned}
& a_{0} x^{n}+a_{1} x^{n-1} y+\cdots a_{n-1} x y^{n-1}+a_{n} y^{n}=c \\
& \left(a_{i}, c \text { rational integers, } i=0,1, \ldots, n ; n>2\right)
\end{aligned}
$$


has only a finite number of (rational) solutions is an amazing discovery. It leaves open the question how to find these solutions and what is their exact number, and one would conjecture that it will remain open for (all) times to come.

The equations considered in this part of the paper are:

i) The equation, known (wrongly) as Pell's equation, namely $x^{2}-m y^{2}= \pm 1$,

ii) The equation $x^{3}+m y^{3}+m^{2} z^{3}-3 m x y z=1$, iii) and

$\left|\begin{array}{lllll}\mathrm{x} & \mathrm{y} & \mathrm{z} & \mathrm{u} & \mathrm{v} \\ \mathrm{mv} & \mathrm{x} & \mathrm{y} & \mathrm{z} & \mathrm{u} \\ \mathrm{mu} & \mathrm{mv} & \mathrm{x} & \mathrm{y} & \mathrm{z} \\ \mathrm{mz} & \mathrm{mu} & \mathrm{mv} & \mathrm{x} & \mathrm{y} \\ \mathrm{my} & \mathrm{mz} & \mathrm{mu} & \mathrm{mv} & \mathrm{x}\end{array}\right|=1$.

Infinitely many solutions of each of these equations will be stated explicitly. Now, it is known that the solutions of Pell's equations is well exploited. Still, we found it necessary to include it here because of the simple method we shall use in solving this equation here, which has such a wide range of application in various branches of exact sciences. Also, we will derive some new combinatorial identities.

Since we are going to use some formulas obtained by the author in some previous papers [1] and [2] we introduce them here

$$
\begin{aligned}
& \left.\begin{array}{l}
A_{0}^{(0)}=1, A_{0}^{(1)}=0, A_{0}^{(n+2)}=A_{0}^{(n)}+2 D A_{0}^{(n+1)} \\
A_{1}^{(0)}=0, A_{1}^{(1)}=1, A_{1}^{(n+2)}=A_{1}^{(n)}+2 D A_{1}^{(n+1)} \\
n=0,1, \ldots
\end{array}\right\} \\
& A_{1}^{(v+l)}=A_{0}^{(v)}+2 D A_{0}^{(v+l)}=A_{1}^{(v+2)} \text {. } \\
& A_{0}^{(2 n)}=\sum_{i=0}^{n-1}\left(\begin{array}{c}
2 n-2-i \\
i
\end{array}\right)(2 D)^{2 n-2-2 i}, n=1,2, \ldots \\
& \text { for } n \Rightarrow 2 n-1 \text {. } \\
& A_{0}^{(2 n+1)}=\sum_{i=0}^{n-1}\left(\begin{array}{c}
2 n-1-i \\
i
\end{array}\right)(2 D)^{2 n-1-2 i}, n=1,2, \ldots \\
& e^{f}=A_{0}^{(f \ell)}+a_{1}^{(0)} A_{0}^{(f \ell+1)}+\cdots+a_{n-1}^{(0)} A_{0}^{(f \ell+n-1)}, f=1,2, \ldots
\end{aligned}
$$

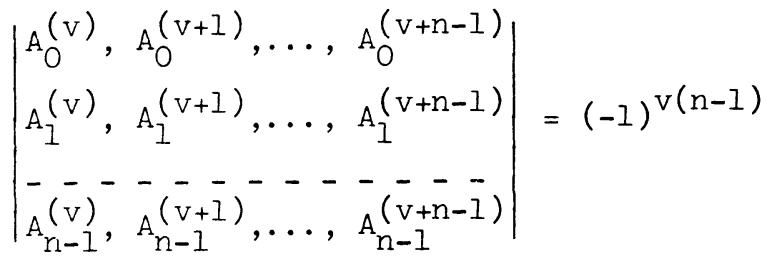

$$
\begin{aligned}
& \left.A_{O}^{(0)}=1, A_{O}^{(1)}=A_{O}^{(2)}=0, A_{O}^{(n+3)}=A_{O}^{(n)}+3 D A_{O}^{(n+1)}+3 D^{2} A_{O}^{(n+2)}\right\}
\end{aligned}
$$




$$
\begin{aligned}
& A_{1}^{(n+2)}=A_{0}^{(n+1)}+3 D A_{0}^{(n+2)} \\
& A_{2}^{(n+2)}=A_{0}^{(n)}+3 D A_{0}^{(n+1)}+3 D^{2} A_{0}^{(n+2)} \\
& \prod_{i=1}^{k} a_{n-1}^{(i)}=A_{0}^{(k)}+a_{1}^{(k)} A_{0}^{(k+1)}+\cdots+a_{n-1}^{(k)} A_{0}^{(k+n-1)} \\
& A_{0}^{(n+3)}=\sum_{3 y_{1}+2 y_{2}+y_{3}=n}\left(\begin{array}{l}
y_{1}+y_{2}+y_{3} \\
y_{1}, y_{2}, y_{3}
\end{array}\right) 3^{y_{2}+y_{3}} D_{2}+2 y_{3} \\
& n=0,1, \ldots ;\left(\begin{array}{l}
0 \\
0
\end{array}\right) \equiv I \\
& A_{0}^{(0)}=I, A_{0}^{(1)}=A_{0}^{(2)}=A_{0}^{(3)}=A_{0}^{(4)}=0 . \\
& A_{0}^{(n+5)}=A_{0}^{(n)}+5 D A_{0}^{(n+1)}+10 D^{2} A_{0}^{(n+2)}+10 D^{3} A_{0}^{(n+3)}+5 D^{4} A_{0}^{(n+4)} \\
& n=0, I, \ldots
\end{aligned}
$$

1. PELL'S EQUATION

Vie denote

$$
\begin{aligned}
& w^{2}=D^{2}+1=m, w=\sqrt{D^{2}+1}, \\
& D \in N, m \text { not a perfect square. }
\end{aligned}
$$

We obtain from $(0.6)$ with $\mathrm{n}=2$,

$$
\begin{aligned}
& \left|\begin{array}{cc}
A_{0}^{(n)} & A_{0}^{(n+1)} \\
A_{1}^{(n)} & \hat{A}_{1}^{(n+1)}
\end{array}\right|=(-1)^{(2-1) n} \\
& n=0,1, \ldots
\end{aligned}
$$

and from $(0.1),(0.2)$

$$
\begin{aligned}
& \left|\begin{array}{ll}
A_{0}^{(n)} & A_{0}^{(n+1)} \\
A_{0}^{(n+1)} & A_{0}^{(n+2)}
\end{array}\right|=\left|\begin{array}{ll}
A_{0}^{(n)} & A_{0}^{(n+1)} \\
A_{0}^{(n+1)} & A_{0}^{(n)}+2 D A_{0}^{(n+1)}
\end{array}\right|= \\
& A_{O}^{(n)^{2}}+2 D A_{O}^{(n)} A_{O}^{(n+1)}-A_{O}^{(n+1)^{2}}=\left(A_{O}^{(n)}+D A_{O}^{(n+1)}\right)^{2}-\left(D^{2}+1\right) A_{O}^{(n+1)^{2}} \text {, } \\
& \left(A_{0}^{(n)}+D A_{0}^{(n+1)}\right)^{2}-m A_{O}^{(n+1)^{2}}=(-1)^{n} \text {. }
\end{aligned}
$$

We have obtained Pell's equations

$$
\begin{aligned}
& x_{n}^{2}-m y_{n}^{2}=(-1)^{n}, \quad n=0,1, \ldots \\
& x_{n}=A_{0}^{(n)}+D A_{0}^{(n+1)}, y_{n}=A_{0}^{(n+1)} \\
& x_{2 n}=A_{0}^{(2 n)}+D A_{0}^{(2 n+1)}, y_{2 n}=A_{0}^{(2 n+1)} \\
& x_{2 n}^{2}-m y_{2 n}^{2}=1 . \\
& x_{2 n+1}=A_{0}^{(2 n+1)}+D A_{0}^{(2 n+2)}, y_{2 n+1}=A_{0}^{(2 n+2)} \\
& x_{2 n+1}^{2}-m y_{2 n+1}^{2}=-1 .
\end{aligned}
$$


Thus we have obtained infinitely many solutions of $x^{2}-m y^{2}= \pm 1$, and, as is known from the theory of continued fractions, these are all solutions of these two equations, the so-called plus and minus cases of Pell's equations.

With (0.3), (0.4), formulas (1.5), (1.6) take the forms

$$
\begin{aligned}
x_{2 n}= & \sum_{i=0}^{n-1}\left(\begin{array}{c}
2 n-2-i \\
i
\end{array}\right)(2 D)^{2 n-2-2 i}+\frac{1}{2}\left(\begin{array}{c}
2 n-1-i \\
i
\end{array}\right)(2 D)^{2 n-2 i}, \\
y_{2 n}= & \sum_{i=0}^{n-1}\left(\begin{array}{c}
2 n-1-i \\
i
\end{array}\right)(2 D)^{2 n-1-2 i}, \\
& x_{2 n}^{2}-m y_{2 n}^{2}=1, n=0,1, \ldots \\
x_{2 n+1}= & \sum_{i=0}^{n-1}\left(\begin{array}{c}
2 n-1-i \\
i
\end{array}\right)(2 D)^{2 n-1-2 i}+\sum_{i=0}^{n}\left(_{i}^{2 n-i}\right)(2 D)^{2 n+1-2 i}, \\
y_{2 n+1}= & \sum_{i=1}^{n}\left(\begin{array}{c}
2 n-i \\
i
\end{array}\right)(2 D)^{2 n-2 i}, \quad n=0,1, \ldots \\
& x_{2 n+1}^{2}-m y_{2 n+1}^{2}=-1 .
\end{aligned}
$$

With the calculations of $A_{0}^{(v)}$ from $C h$. 0 , we have

$$
\begin{aligned}
& \mathrm{x}_{0}=\mathrm{A}_{\mathrm{O}}^{(0)}+\mathrm{DA}_{\mathrm{O}}^{(1)}=\mathrm{A}_{\mathrm{O}}^{(0)}=1 ; \mathrm{A}_{\mathrm{O}}^{(1)}=\mathrm{y}_{1}=0 \text {, } \\
& x_{0}^{2}=m y_{0}^{2}=1^{2}-\left(D^{2}+1\right) \cdot 0=1 \text {. } \\
& \mathrm{x}_{1}=\mathrm{A}_{0}^{(1)}+\mathrm{DA}_{0}^{(2)}=\mathrm{D} ; \mathrm{y}_{1}=\mathrm{A}_{0}^{(2)}=1 \text {, } \\
& x_{1}^{2}-m y_{1}^{2}=D^{2}-\left(D^{2}+1\right) \cdot 1=-1 \text {. } \\
& \mathrm{x}_{2}=\mathrm{A}_{0}^{(2)}+\mathrm{DA}_{\mathrm{O}}^{(3)}=1+2 \mathrm{D}^{2} ; \mathrm{y}_{2}=2 \mathrm{D} \text {, } \\
& x_{2}^{2}-m y_{2}^{2}=\left(1+2 D^{2}\right)^{2}+\left(1+D^{2}\right) \cdot 4 D^{2}=1 \text {. } \\
& x_{3}=A_{0}^{(3)}+D A_{0}^{(4)}=3 D+4 D^{3} ; y_{3}=1+4 D^{2} \text {, } \\
& x_{3}^{2}-m y_{3}^{2}=\left(3 D+4 D^{3}\right)^{2}-\left(1+D^{2}\right)\left(1+4 D^{2}\right)^{2}=-1 \text {, } \\
& x_{4}=1+8 D^{2}+8 D^{4} ; y_{4}=4 D+8 D^{3} \text {, } \\
& x_{4}^{4}-m y_{4}^{2}=\left(1+8 D^{2}+8 D^{4}\right)^{2}-\left(1+D^{2}\right)\left(4 D+8 D^{3}\right)^{2}=1 .
\end{aligned}
$$

2. UNITS IN $Q(w), w=\sqrt{D^{2}+1}$

It is clear that

$$
e=w+D
$$

is a unit in $Q(w)$. For $e$ is an integer, and $e^{-1}=w-D$, an integer.

The ACF [l] of $a^{(O)}=w+D$ is purely periodic with length of its primitive period $\ell=1$; hence we have from formula (0.5)

$$
e^{n}=(w+D)^{n}=A_{0}^{(n)}+(w+D) A_{0}^{(n+1)} \text {. }
$$


From (2.2) we get an interesting combinatorial identity

$$
\begin{aligned}
& (w+D)^{2 n}=A_{0}^{(2 n)}+(w+D) A_{0}^{(2 n+1)} \\
& (w+D)^{2 n}=A_{0}^{(2 n)}+D A_{0}^{(2 n+1)}+w_{O}^{(2 n+1)},
\end{aligned}
$$

hence from ( 1.5$)$

$$
(\mathrm{w}+\mathrm{D})^{2 \mathrm{n}}=\mathrm{x}_{2 \mathrm{n}}+\mathrm{y}_{2 \mathrm{n}} \mathrm{w} \text {. }
$$

With $w^{2}=D^{2}+l=m$, the reader will easily verify the formulas

$$
(w+D)^{2 n}=\left(\sum_{i=0}^{n}\left(\begin{array}{l}
2 n \\
2 i
\end{array}\right) D^{2 i} m^{n-i}\right)+\left(\sum_{i=0}^{n-1}\left(\begin{array}{c}
2 n \\
2 i+1
\end{array}\right) D^{2 i+1} m^{n-1-i}\right) w .
$$

From (2.3) and (2.4), and using the expressions for $x_{2 n}$ and $y_{2 n}$ from the previous paragraph, we obtain the combinatorial identities

$$
\begin{aligned}
& \sum_{i=0}^{n-1}\left[\left(\begin{array}{c}
2 n-2-i \\
i
\end{array}\right)(2 D)^{2 n-2-2 i}+\frac{1}{2}\left(\begin{array}{c}
2 n-1-i \\
i
\end{array}\right)(2 D)^{2 n-2 i}\right] \\
& \quad=\sum_{i=0}^{n}\left(\begin{array}{l}
2 n \\
2 i
\end{array}\right) D^{2 i}\left(D^{2}+1\right)^{n-i} . \\
& \sum_{i=0}^{n-1}\left(\begin{array}{c}
2 n-1-i \\
i
\end{array}\right)(2 D)^{2 n-1-2 i}=\sum_{i=0}^{n-1}\left(\begin{array}{c}
2 n \\
2 i+1
\end{array}\right) D^{2 i+1}\left(D^{2}+1\right)^{n-1-i} .
\end{aligned}
$$

Similar identities are obtainable from

$$
(w+D)^{2 n+1}=x_{2 n+1}+y_{2 n+1} w .
$$

3. THE CUBIC DIOPHANTINE EQUATIONS

We shall need formulas $(0.6)$, $(0.7),(0.8)$, (0.9) for $n=3$, viz.

$$
\begin{aligned}
& \left|\begin{array}{ccc}
A_{0}^{(n+1)} & A_{0}^{(n+2)} & A_{0}^{(n+3)} \\
A_{1}^{(n+1)} & A_{1}^{(n+2)} & A_{1}^{(n+3)} \\
A_{2}^{(n+1)} & A_{2}^{(n+2)} & A_{2}^{(n+3)}
\end{array}\right|=1 \\
& A_{O}^{(0)}=1, \quad A_{O}^{(1)}=A_{O}^{(2)}=0 \text {, } \\
& A_{O}^{(n+3)}=A_{O}^{(n)}+3 D A_{0}^{(n+1)}+3 D^{2} A_{0}^{(n+2)} \text {, } \\
& A_{1}^{(n+3)}=A_{0}^{(n+2)}+3 A_{0}^{(n+3)} \\
& A_{2}^{(n+3)}=A_{0}^{(n+1)}+3 D A_{0}^{(n+2)}+3 D^{2} A_{0}^{(n+3)} \text {. }
\end{aligned}
$$

Substituting in (3.1) the values for $A_{1}^{(i)}, A_{2}^{(i)}, i=n+3$ from (3.2), we obtain, after simple rearrangements

$$
I=\left|\begin{array}{lll}
A_{O}^{(n+1)} & A_{O}^{(n+2)} & A_{O}^{(n+3)} \\
A_{O}^{(n)}+3 D A_{O}^{(n+1)} & A_{O}^{(n+1)}+3 D A_{O}^{(n+2)} & A_{O}^{(n+2)}+3 D A_{O}^{(n+3)} \\
A_{O}^{(n+1)}+3 D A_{O}^{(n)}+3 D^{2} A_{O}^{(n+1)} & A_{O}^{(n)}+3 D A_{O}^{(n+1)}+3 D^{2} A_{O}^{(n+2)} & A_{O}^{n+1}+3 D A_{O}^{n+2}+3 D^{2} A_{O}^{(n+3)}
\end{array}\right|
$$




$$
=\left|\begin{array}{lll}
A_{0}^{(n+1)} & A_{0}^{(n+2)} & A_{0}^{(n+3)} \\
A_{0}^{(n)} & A_{0}^{(n+1)} & A_{0}^{(n+2)} \\
A_{0}^{(n-1)} & A_{0}^{(n)} & A_{0}^{(n+1)}
\end{array}\right| .
$$

We now denote

$$
\begin{gathered}
x=A_{0}^{(n-1)}, y=A_{0}^{(n)}, z=A_{0}^{(n+1)} \\
n=1,2, \ldots .
\end{gathered}
$$

and obtain for the above determinant

$$
I=\left|\begin{array}{ccc}
z & x+3 D y+3 D^{2} z & y+3 D z+3 D^{2} A_{0}^{(n+3)} \\
y & z & A_{0}^{(n+2)} \\
x & y & z
\end{array}\right| .
$$

Subtracting from the first row the 3D multiple of the third and the $3 D^{2}$ of the second, we obtain,

$$
\left|\begin{array}{ccc}
2-3 D x-3 D^{2} y & x & y \\
y & z & x+3 D y+3 D^{2} z \\
x & y & z
\end{array}\right|=1
$$

We leave it to the reader to expand the determinant in (3.4) to obtain the Diophantine equation of the third degree as

$$
\left.\begin{array}{rl}
x^{3} & +\left(9 D^{3}+1\right) y^{3}+z^{3}+\left(9 D^{3}-3\right) x y z+6 D x^{2} y+3 D^{2} x^{2} z \\
& +12 D^{2} y^{2} x+\left(9 D^{4}-3 D\right) y^{2} z-3 D z^{2} x-6 D^{2} z^{2} y=1
\end{array}\right\}
$$

Even for $D=1$, equation (3.5) has a complicated form as

$$
\begin{aligned}
x^{3} & +10 y^{3}+z^{3}+6 x y z+6 x^{2} y+3 x^{2} z+12 y^{2} x \\
& +6 y^{2} z-3 z^{2} x-6 z^{2} y=1
\end{aligned}
$$
$A_{0}^{(n+2)}, n=0,1, \ldots$. [2] we have calculated the solution triples $A_{0}^{(n)}, A_{0}^{(n+1)}$,

$$
\begin{aligned}
(x, y, z)= & (1,0,0),(0,0,1),(0,1,3),(1,3,12), \\
& (3,12,46),(12,46,177) .
\end{aligned}
$$

We shall check the solution

$$
\left(\mathrm{x}_{3}, \mathrm{y}_{3}, \mathrm{z}_{3}\right)=(1,3,12) \text {. }
$$

Substituting these values in (3.6), we obtain $1+270+1728+216+$ $18+36+108+648-432-2592=1,3025-3024=1$.

For larger values of $D$ and $n$ the verification of (3.5) is only possible by computer, and without knowing (3.3) even a computer would have its problems.

As we shall soon see, there is a much simpler third degree Diophantine equation which can be regarded as, and indeed in a certain case represents, a generalization of Pell's equation to the third degree. 
4. UNITS IN THE CUBIC FIELD

As we have seen in $[1]$, the ACF of the vector a ${ }^{(0)} \in E_{3}$, with $w=\sqrt[3]{D^{3}+1}, D \in N, a^{(0)}=\left(w+2 D, w^{2}+D w+D^{2}\right)$, is purely periodic with length of primitive period $\ell=1$. Hence, by theorem 2 in [2] and formula $(0.10)$

$$
e=w^{2}+D w+D^{2}
$$

is a unit in $Q(w)$, and

$$
\begin{gathered}
e^{v}=A_{O}^{(v)}+(w+2 D) A_{O}^{(v+1)}+\left(w^{2}+D w+D^{2}\right) A_{O}^{(v+2)} \\
v=0,1, \ldots .
\end{gathered}
$$

Thus

$$
\begin{aligned}
\left(w^{2}+D w+D^{2}\right)^{v}= & A_{0}^{(v)}+2 D A_{0}^{(v+1)}+D^{2} A_{0}^{(v+2)} \\
& +\left(A_{0}^{(v+1)}+D A_{0}^{(v+2)}\right) w+D^{2} A_{0}^{(v+2)} w^{2} .
\end{aligned}
$$

We shall find the field equation of the expressions (1.3) in $Q(w)$.

We denote

$$
\begin{aligned}
& x_{v}=A_{O}^{(v)}+2 D A_{O}^{(v+1)}+D^{2} A_{O}^{(j+2)}, \\
& y_{v}=A_{O}^{(v+1)}+D A_{O}^{(v+2)} \\
& z_{v}=A_{O}^{(v+2)},
\end{aligned}
$$

and have

$$
\begin{gathered}
\mathrm{e}^{\mathrm{v}}=\mathrm{x}_{\mathrm{v}}+\mathrm{y}_{\mathrm{v}} \mathrm{w}+\mathrm{z}_{\mathrm{v}} \mathrm{w}^{2} \\
\mathrm{we}^{\mathrm{v}}=\mathrm{m} \mathrm{z}_{\mathrm{v}}+\mathrm{x}_{\mathrm{v}} \mathrm{w}+\mathrm{y}_{\mathrm{v}} \mathrm{w}^{2} \\
\mathrm{w}^{2} \mathrm{e}^{\mathrm{v}}=\mathrm{my} \mathrm{y}_{\mathrm{v}}+\mathrm{m} \mathrm{z}_{\mathrm{v}} \mathrm{w}+\mathrm{x}_{\mathrm{v}} \mathrm{w}^{2} \\
\mathrm{~m}=\mathrm{w}^{3}=\mathrm{D}^{3}+1 .
\end{gathered}
$$

Hence

$$
\left|\begin{array}{ccc}
\mathrm{x}_{\mathrm{v}} & \mathrm{y}_{\mathrm{v}} & \mathrm{z}_{\mathrm{v}} \\
\mathrm{m} \mathrm{z}_{\mathrm{v}} & \mathrm{x}_{\mathrm{v}} & \mathrm{y}_{\mathrm{v}} \\
\mathrm{my} \mathrm{v}_{\mathrm{v}} & \mathrm{mz} \mathrm{v}_{\mathrm{v}} & \mathrm{x}_{\mathrm{v}}
\end{array}\right|=1,
$$

since $N(e)=1$, as the reader will easily verify.

Expanding the determinant in (4.6), we obtain

$$
\begin{aligned}
x_{v}^{3}+m y_{v}^{3}+m^{2} z_{v}^{3}-3 m x_{v} y_{v} z_{v} & =1 \\
x_{v}, y_{v}, z_{v} & \text { from }(4.4), v=0,1, \ldots .
\end{aligned}
$$

The Diophantine equation

$$
x^{3}+m y^{3}+m^{2} z^{3}-3 m x y z=1
$$

is indeed Pell's equation generalized to the third dimension. It is simpler compared with (3.6) and it has as solutions (4.4).

We shall verify formula (4.5), first line for $v=1,2$. We have, from (4.4), 


$$
\begin{aligned}
x_{1} & =A_{0}^{(1)}+2 D A_{0}^{(2)}+D^{2} A_{O}^{(3)}=D^{2}, \\
y_{1} & =A_{O}^{(2)}+D A_{O}^{(3)}=D, \\
z_{1} & =A_{0}^{(3)}=1 . \\
\left(D^{2}\right)^{3}+ & \left(D^{3}+1\right) D^{3}+\left(D^{3}+1\right)^{2} \cdot 1-3\left(D^{3}+1\right) D^{2} \cdot D= \\
D^{6} & +D^{6}+D^{3}+D^{6}+2 D^{3}+1-3 D^{6}-3 D^{3}=1 ; \\
x_{2} & =A_{0}^{(2)}+2 D A_{O}^{(3)}+D^{2} A_{O}^{(4)} \\
x_{2} & =2 D+D^{2} \cdot 3 D^{2}=2 D+3 D^{4}, \\
y_{2} & =A_{O}^{(3)}+D A_{O}^{(4)}=1+3 D^{3} \\
z_{2} & =A_{0}^{(4)}=3 D^{2} .
\end{aligned}
$$

We obtain substituting $\left(\mathrm{x}_{2}, \mathrm{y}_{2}, \mathrm{z}_{2}\right)$ in (4.7)

$$
1+18 D^{3}+99 D^{6}+162 D^{9}+81 D^{12}-3\left(6 D^{3}+33 D^{6}+54 D^{9}+27 D^{12}\right)=1 .
$$

We shall now extract a few interesting identities from Formula (4.3). We have, by the binomial theorem,

$$
\begin{aligned}
&\left(w^{2}+D w+D^{2}\right)^{3 n}=\sum_{i=0}^{3 n}\left(w^{2}\right)^{2 n-i}\left(D w+D^{2}\right)^{i}= \\
&=\sum_{i=0}^{3 n}\left(\begin{array}{c}
3 n \\
i
\end{array}\right) w^{6 n-2 i} \sum_{j=0}^{i}\left(\begin{array}{c}
i \\
j
\end{array}\right)(D w)^{i-j} D^{2 j}= \\
&=\sum_{i=0}^{3 n}\left(\begin{array}{c}
3 n \\
i
\end{array}\right) w^{6 n-2 i}\left(\begin{array}{c}
i \\
j
\end{array}\right) D^{i+j} w^{i-j}= \\
& j=0,1, \ldots, i \\
&=\sum_{i=0}^{3 n}\left(\begin{array}{c}
3 n \\
i
\end{array}\right)\left(\begin{array}{c}
i \\
j
\end{array}\right) w^{6 n-(i+j)} D^{i+j} . \\
& j=0,1, \ldots, i
\end{aligned}
$$

In the $\operatorname{sum} \sum\left(\begin{array}{c}3 n \\ i\end{array}\right)\left(\begin{array}{c}i \\ j\end{array}\right) w^{6 n-(i+j)} D^{i+j}, i=0,1, \ldots, 3 n ; j=0,1, \ldots, i$; we want to find the coefficient of powers of $w^{3 n}$, so that since $\mathrm{w}^{3}=\mathrm{m}=\mathrm{D}^{3}+3$, this sum becomes rational. For this purpose we have to set $i+j \equiv O(3)$ and obtain

$$
\begin{aligned}
& \left(w^{2}+D w+D^{2}\right)^{3 n}= \\
& \quad \sum_{i+j=3 s \leq 6 n}\left(\begin{array}{c}
3 n \\
i
\end{array}\right)\left(\begin{array}{c}
i \\
j
\end{array}\right) w^{6 n-3 s} D^{3 s} \\
& 0 \leq 0,1, \ldots, 2 n \\
& 0 \leq j \leq i \leq 3 n
\end{aligned}
$$

(4.8) is an appealing formula for the expression $\left(w^{2}+D w+D^{2}\right)^{3 n}$, though this expression could also be calculated by the multinomial theorem. We have, in order to illustrate its application; $n=1, s=0,1,2$. 


$$
\begin{aligned}
& s=0 ; i=j=0 ; \\
& s=1 ; i=2, j=I ; i=3, j=0 ; \\
& s=2 ; i=3, j=3 . \quad(i \leq 3) .
\end{aligned}
$$

Hence we have for the rational part of $e^{3}$ :

$$
\begin{aligned}
\left(w^{2}+D w+D^{2}\right)^{3}= & \left(\begin{array}{l}
3 \\
0
\end{array}\right)\left(\begin{array}{l}
0 \\
0
\end{array}\right) w^{6}+\left(\begin{array}{l}
3 \\
2
\end{array}\right)\left(\begin{array}{l}
2 \\
1
\end{array}\right) w^{3} D^{3}+\left(\begin{array}{l}
3 \\
3
\end{array}\right)\left(\begin{array}{l}
3 \\
0
\end{array}\right) w^{3} D^{3}+ \\
& +\left(\begin{array}{l}
3 \\
3
\end{array}\right)\left(\begin{array}{l}
3 \\
3
\end{array}\right) D^{6}=w^{6}+7 D^{3} w^{3}+D^{6}, \\
{\left[w^{2}+\left(D w+D^{2}\right)\right]^{3}=} & w^{6}+3 w^{4}\left(D w+D^{2}\right)+3 w^{2}\left(D w+D^{2}\right)^{2}+\left(D w+D^{2}\right)^{3}= \\
= & w^{6}+3 D w^{5}+3 D^{2} w^{4}+3 D^{2} w^{4}+6 D^{3} w^{3}+ \\
& +3 w^{2} D^{4}+D^{3} w^{3}+3 D^{4} w^{2}+3 D^{5} w+D^{6} .
\end{aligned}
$$

The rational members of this sum are $w^{6}+6 D^{3} w^{3}+D^{3} w^{3}+D^{6}$, as was calculated above, with $\mathrm{w}^{3}=\mathrm{m}=\mathrm{D}^{3}+1$. The formula $(4.8)$ is easily applicable since there is no difficulty to solve the linear equations $i+j=3 \mathrm{~s}$.

We shall still find the rational part of $e^{6}=\left(w^{2}+D w+D^{2}\right)^{6}$. By formula (4.8), with

$$
\begin{aligned}
& \mathrm{n}=2, \mathrm{~s}=0,1,2,3,4 ; i+j=3 \mathrm{~s}, j \leq i \leq 6 \text {; } \\
& s=0 ; i=j=0 \text {; } \\
& s=1 ; i=3, j=0 \text {, } \\
& i=2, j=1 \text {; } \\
& s=2 ; i=6, j=0 \text {, } \\
& i=5, j=1 \text {, } \\
& i=4, j=2 \text {, } \\
& i=3, j=3 \text {; } \\
& s=3 ; i=6, j=3 \text {, } \\
& i=5, j=4 \text {; } \\
& s=4 ; i=6, j=6 \text {; }
\end{aligned}
$$

we obtain

$$
\begin{aligned}
& \left(\begin{array}{l}
6 \\
0
\end{array}\right)\left(\begin{array}{l}
0 \\
0
\end{array}\right) \mathrm{w}^{12}+\left[\left(\begin{array}{l}
6 \\
3
\end{array}\right)\left(\begin{array}{l}
3 \\
0
\end{array}\right)+\left(\begin{array}{l}
6 \\
2
\end{array}\right)\left(\begin{array}{l}
2 \\
1
\end{array}\right)\right] \mathrm{w}^{9} \mathrm{D}^{3}+ \\
& +\left[\left(\begin{array}{l}
6 \\
6
\end{array}\right)\left(\begin{array}{l}
6 \\
0
\end{array}\right)+\left(\begin{array}{l}
6 \\
5
\end{array}\right)\left(\begin{array}{l}
5 \\
1
\end{array}\right)+\left(\begin{array}{l}
6 \\
4
\end{array}\right)\left(\begin{array}{l}
4 \\
2
\end{array}\right)+\left(\begin{array}{l}
6 \\
3
\end{array}\right)\left(\begin{array}{l}
3 \\
3
\end{array}\right)\right] \mathrm{w}^{6} \mathrm{D}^{6}+ \\
& +\left[\left(\begin{array}{l}
6 \\
6
\end{array}\right)\left(\begin{array}{l}
6 \\
3
\end{array}\right)+\left(\begin{array}{l}
6 \\
5
\end{array}\right)\left(\begin{array}{l}
5 \\
4
\end{array}\right)\right] \mathrm{w}^{3} \mathrm{D}^{9}+\left(\begin{array}{l}
6 \\
6
\end{array}\right)\left(\begin{array}{l}
6 \\
6
\end{array}\right) \mathrm{D}^{12}= \\
& =\mathrm{w}^{12}+50 \mathrm{w}^{9} \mathrm{D}^{3}+141 \mathrm{w}^{6} \mathrm{D}^{6}+50 \mathrm{w}^{3} \mathrm{D}^{3}+\mathrm{D}^{12}= \\
& =\mathrm{m}^{4}+50 \mathrm{~m}^{3} \mathrm{D}^{3}+141 \mathrm{~m}^{2} \mathrm{D}^{6}+50 \mathrm{~m} \mathrm{D}^{9}+\mathrm{D}^{12}, \\
& \quad \mathrm{~m}=\mathrm{D}^{3}+1=\mathrm{w}^{3} .
\end{aligned}
$$

We thus have the final result, viz. The rational part of $e^{3 n}=$ $\left(w^{2}+D w+D^{2}\right)^{3 n}$ equals

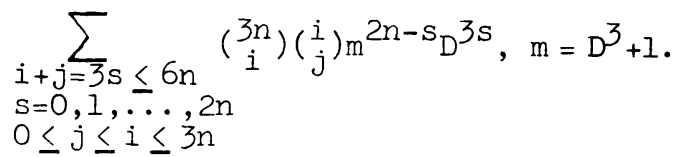


We shall now find the coefficient of $w$ in

$$
\sum_{\substack{i=0 \\
j=0,1, \ldots, i}}^{3 n}\left(\begin{array}{c}
3 n \\
i
\end{array}\right)\left(\begin{array}{c}
i \\
j
\end{array}\right) w^{6 n-(i+j)} D^{i+j}
$$

and demand, to this end,

$$
\begin{aligned}
& 6 n-(i+j) \equiv 1(\bmod 3), \\
& i+j \equiv 2(3), i+j=3 s+2 \\
& s=0,1, \ldots, 2 n-1,
\end{aligned}
$$

and obtain thus, that this coefficient equals

$$
\begin{aligned}
& \sum_{\substack{i+j=3 s+2 \\
s=0,1, \ldots, 2 n-1, 0 \leq j \leq i \leq 3 n .}}\left(\begin{array}{c}
3 n \\
i
\end{array}\right)\left(\begin{array}{l}
i \\
j
\end{array}\right) w^{6 n-(3 s+2)} D^{3 s+2} \\
&
\end{aligned}
$$

But $w^{6 n-(3 s+2)}=w^{6 n-(3 s+3)+1}=$

$$
\begin{aligned}
= & \mathrm{w}^{3[2 n-(s+1)]+1}=\mathrm{m}^{2 n-(s+1)} \mathrm{w}, \\
& \mathrm{m}=\mathrm{w}^{3}=\mathrm{D}^{3}+1 .
\end{aligned}
$$

Hence,

The coefficient of $w$ in $\left(w^{2}+D w+D^{2}\right)^{3 n}$ equals

$$
\begin{aligned}
& \sum_{\substack{i+j=3 s+2 ; \\
s=0,1, \ldots, 2 n-1}} D^{3 s+2} m^{2 n-s-1}\left(\begin{array}{c}
3 n \\
i
\end{array}\right)\left(\begin{array}{l}
i \\
j
\end{array}\right) . \\
& 0 \leq j \leq i \leq 3 n
\end{aligned}
$$

Illustration of $(4.12)$ :

$$
\begin{aligned}
& n=1 ; s=0,1 ; s=0 ; i=2, j=0 ; i=1, j=1 ; \\
& s=1 ; i=3, j=2 .
\end{aligned}
$$

The coefficient of $w$ in the expansion of $\left(w^{2}+D w+D^{2}\right)^{3}$ equals

$$
D^{2} m\left[\left(\begin{array}{l}
3 \\
2
\end{array}\right)\left(\begin{array}{l}
2 \\
0
\end{array}\right)+\left(\begin{array}{l}
3 \\
1
\end{array}\right)\left(\begin{array}{l}
1 \\
1
\end{array}\right)\right]+D^{5}\left[\left(\begin{array}{l}
3 \\
3
\end{array}\right)\left(\begin{array}{l}
3 \\
2
\end{array}\right)\right]=6 D^{2}+3 D^{5},
$$

as the reader can verify.

$$
\begin{aligned}
n=2 ; & s=0,1,2,3 ; \quad 3 n=6 \geq i . \\
& s=0 ; i=2, j=0 ; i=1, j=1 ; \\
& s=1 ; i=5, j=0 ; i=4, j=1 ; i=3, j=2 ; \\
& s=2 ; i=6, j=1 ; i=5, j=2 ; i=4, j=3 ; \\
& s=3 ; i=6, j=5 .
\end{aligned}
$$

The coefficient of $w$ in $\left(w^{2}+D w+D^{2}\right)^{6}$ equals

$$
\begin{aligned}
& {\left[\left(\begin{array}{l}
6 \\
2
\end{array}\right)\left(\begin{array}{l}
2 \\
0
\end{array}\right)+\left(\begin{array}{l}
6 \\
l
\end{array}\right)\left(\begin{array}{l}
1 \\
l
\end{array}\right)\right] D^{2} m^{3}+\left[\left(\begin{array}{l}
6 \\
5
\end{array}\right)\left(\begin{array}{l}
5 \\
0
\end{array}\right)+\left(\begin{array}{l}
6 \\
l
\end{array}\right)\left(\begin{array}{l}
1 \\
l
\end{array}\right)\right] D^{5} m^{2}+} \\
& +\left[\left(\begin{array}{l}
6 \\
6
\end{array}\right)\left(\begin{array}{l}
6 \\
l
\end{array}\right)+\left(\begin{array}{l}
6 \\
5
\end{array}\right)\left(\begin{array}{l}
5 \\
2
\end{array}\right)+\left(\begin{array}{l}
6 \\
4
\end{array}\right)\left(\begin{array}{l}
4 \\
3
\end{array}\right)\right] D^{8} m+\left[\left(\begin{array}{l}
6 \\
0
\end{array}\right)\left(\begin{array}{l}
6 \\
5
\end{array}\right)\right] D^{11}= \\
& =21 D^{2} m^{3}+12 D^{5} m^{2}+126 D^{8} m+6 D^{11} .
\end{aligned}
$$

The reader will now prove without any difficulty that: 
The coefficient of $w^{2}$ in $\left(w^{2}+D w+D^{2}\right)^{3 n}$ equals

$$
\begin{aligned}
& \sum_{\substack{i+j=3 s+1 ; \\
s=0,1, \ldots, 2 n-1, 0 \leq j \leq i \leq 3 n}}\left(\begin{array}{c}
3 n \\
i
\end{array}\right)\left(\begin{array}{c}
i \\
j
\end{array}\right) D^{3 s+1} m^{2 n-s-1} \\
&
\end{aligned}
$$

But by (4.3) we have

$$
\begin{aligned}
\left(w^{2}+D w+D^{2}\right)^{3 n}= & {\left[A_{0}^{(3 n)}+2 D A_{O}^{(3 n+1)}+D^{2} A_{O}^{(3 n+2)}\right]+} \\
& +\left[A_{O}^{(3 n+1)}+D A_{O}^{(3 n+2)}\right] w+D^{2} A_{O}^{(3 n+2)} w^{2} .
\end{aligned}
$$

With (4.9), (4.12), (4.13) we obtain the identities

$$
\left.\begin{array}{l}
\sum_{\substack{i+j=3 s \leq 6 n \\
0 \leq j \leq i \leq 3 n}}\left(\begin{array}{c}
3 n \\
i
\end{array}\right)\left(\begin{array}{c}
i \\
j
\end{array}\right) m^{2 n-s} D^{3 s}=A_{0}^{(3 n)}+2 D A_{0}^{(3 n+1)}+D^{2} A_{0}^{(3 n+2)} .
\end{array}\right\}
$$

If we substitute in (4.14), (4.14a), (4.14b) the values of $A_{0}^{(3 n)}$, $A_{0}(3 n+1), A_{O}^{(3 n+2)}$, we indeed arrive at some new combinatorial identities. We proceed to obtain further identities for the third dimension.

5. MORE IDENTITIES

$$
\begin{aligned}
& \text { We return to formula (4.2) } \\
& \left(w^{2}+D w+D^{2}\right)^{v}=A_{O}^{(v)}+(w+2 D) A_{O}^{(v+l)}+\left(w^{2}+D w+D^{2}\right) A_{O}^{(v+2)}
\end{aligned}
$$

and have with $(w-D)\left(w^{2}+D w+D^{2}\right)=1$,

$$
(w-D)^{v}=\frac{1}{A_{0}^{(v)}+(w+2 D) A_{0}^{(v+1)}+\left(w^{2}+D w+D^{2}\right) A_{0}^{(v+2)}}
$$

We want to rationalize the denominator in (5.1) so that

$$
\left[A_{0}^{(v)}+(w+2 D) A_{0}^{(v+1)}+\left(w^{2}+D w+D^{2}\right) A_{0}^{(v+2)}\right]\left(a+b w+c w^{2}\right)=1 .
$$

We obtain, with $\mathrm{a}, \mathrm{b}, \mathrm{c}$ rationals,

$$
\begin{aligned}
& \left(A_{0}^{(v)}+2 D A_{0}^{(v+1)}+D^{2} A_{0}^{(v+2)}\right) a+m A_{0}^{(v+2)} b+\left(A_{0}^{(v+1)}+D A_{0}^{(v+2)}\right) m c=1, \\
& \left(A_{0}^{(v+1)}+D A_{0}^{(v+2)}\right) a+\left(A_{0}^{(v)}+2 D A_{0}^{(v+l)}+D^{2} A_{O}^{(v+2)}\right) b+m A_{O}^{(v+2)} c=0, \\
& A_{O}^{(v+2)} a+\left(A_{O}^{(v+1)}+D A_{O}^{(v+2)}\right) b+\left(A_{O}^{(v)}+2 D A_{O}^{(v+1)}+D^{2} A_{O}^{(v+2)}\right) c=0 .
\end{aligned}
$$

The determinant of this system of equations (5.3) equals, with 


$$
\begin{gathered}
x_{v}=A_{O}^{(v)}+2 D A_{O}^{(v+1)}+D^{2} A_{O}^{(v+2)} ; y_{v}=A_{O}^{(v+1)}+D A_{O}^{(v+2)} ; z_{v}=A_{O}^{(v+2)} ; \\
\left|\begin{array}{ccc}
x_{v} & m z_{v} & m y_{v} \\
y_{v} & x_{v} & m z_{v} \\
z_{v} & y_{v} & x_{v}
\end{array}\right|=x_{v}^{3}+m y_{v}^{3}+m^{2} z_{v}^{3}-3 m x_{v} y_{v} z_{v}=1 .
\end{gathered}
$$

Hence we obtain from (5.3)

$$
\begin{aligned}
& a=\left|\begin{array}{ccc}
I & m z_{v} & m y_{v} \\
0 & x_{v} & m z_{v} \\
0 & y_{v} & x_{v}
\end{array}\right|=x_{v}^{2}-m y_{v} z_{v} \\
& b=\left|\begin{array}{ccc}
x_{v} & I & m y_{v} \\
y_{v} & 0 & m z_{v} \\
z_{v} & 0 & x_{v}
\end{array}\right|=m z_{v}^{2}-x_{v} y_{v} \\
& c=\left|\begin{array}{ccc}
x_{v} & m z_{v} & 1 \\
y_{v} & x_{v} & 0 \\
z_{v} & y_{v} & 0
\end{array}\right|=y_{v}^{2}-x_{v} z_{v} .
\end{aligned}
$$

Thus we have obtained the identity.

$$
\begin{aligned}
& (w-D)^{v}=x_{v}^{2}-m y_{v} z_{v}+\left(m z_{v}^{2}-x_{v} y_{v}\right) w+\left(y_{v}^{2}-x_{v} z_{v}\right) w^{2} \text { or } \\
& (w-D)^{3 v}=x_{3 v}^{2}-m_{3 v} z_{3 v}+\left(m z_{3 v}^{2}-x_{3 v} y_{3 v}\right) w+\left(y_{3 v}^{2}-x_{3 v} z_{3 v}\right) w^{2} .
\end{aligned}
$$

Expanding $(3-D)^{3 v}$, we obtain, with $w^{3}=m=\left(D^{3}+I\right)$

$$
\begin{aligned}
(w-D)^{3 v}= & \sum_{i=0}^{v}(-1)^{i}\left(\begin{array}{c}
3 v \\
3 i
\end{array}\right) m^{v-i} D^{3 i}+ \\
& +\left(\sum_{i=0}^{v-1}(-1)^{i}\left(\begin{array}{c}
3 v \\
3 i+2
\end{array}\right) m^{v-1-i} D^{3 i+2}\right) w+ \\
& +\left(\sum_{i=0}^{v-1}(-1)^{i+1}\left(\begin{array}{c}
3 v \\
3 i+1
\end{array}\right) m^{v-1-i} D^{3 i+1}\right) w^{2} .
\end{aligned}
$$

With (5.4), (5.5) we obtain some new identities

$$
\begin{aligned}
& \sum_{i=0}^{v}(-1)^{i}\left(\begin{array}{c}
3 v \\
3 i
\end{array}\right) m^{v-i} D^{3 i}=x_{3 v}^{2}-m y_{3 v} z_{3 v} ; \\
& \sum_{i=0}^{v-1}(-1)^{i}\left(\begin{array}{c}
3 v \\
3 i+2
\end{array}\right) m^{v-1-i} D^{3 i+2}=m z_{3 v}^{2}-x_{3 v} y_{3 v} ; \\
& \sum_{i=0}^{v-1}(-1)^{i+1}\left(\begin{array}{c}
3 v \\
3 i+1
\end{array}\right) m^{v-1-i} D^{3 i+1}=y_{3 v}^{2}-x_{3 v} z_{3 v} ; \\
& v=0,1, \ldots ; x_{v}, y_{v}, z_{v} \text { from }(4.4) .
\end{aligned}
$$


Substituting for $\mathrm{x}_{\mathrm{v}}, \mathrm{y}_{\mathrm{v}}, \mathrm{z}_{\mathrm{v}}$ the values from (4.4), and the values of $A^{(v)}$ from (0.1I) the identities (5.6) take the form

6. FIFTH DEGREE DIOPHANTINE EQUATIONS

We return to formula (0.6) with $n=5$, and obtain

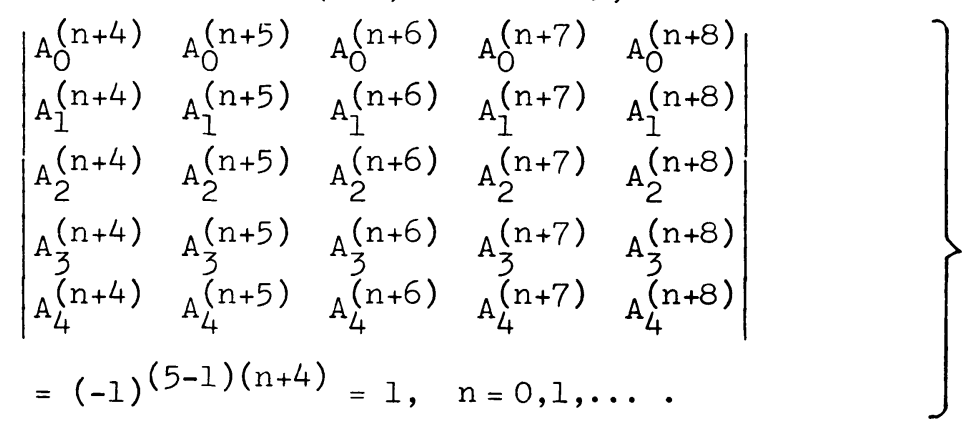

Substituting for $A_{i}^{(v)}, i=1,2,3,4 ; v=n+4, \ldots, n+8$; their representation as forms of $A_{0}^{(n+j)^{i}}, j=0,1,2,3,4$, we obtain the matrix equality.

$$
\left|\begin{array}{lllll}
A_{0}^{(n+4)} & A_{0}^{(n+5)} & A_{0}^{(n+6)} & A_{0}^{(n+7)} & A_{0}^{(n+8)} \\
A_{0}^{(n+3)} & A_{0}^{(n+4)} & A_{0}^{(n+5)} & A_{0}^{(n+6)} & A_{0}^{(n+7)} \\
A_{0}^{(n+2)} & A_{0}^{(n+3)} & A_{0}^{(n+4)} & A_{0}^{(n+5)} & A_{0}^{(n+6)} \\
A_{0}^{(n+1)} & A_{0}^{(n+2)} & A_{0}^{(n+3)} & A_{0}^{(n+4)} & A_{0}^{(n+5)} \\
A_{0}^{(n)} & A_{0}^{(n+1)} & A_{0}^{(n+2)} & A_{0}^{(n+3)} & A_{0}^{(n+4)}
\end{array}\right|=1 .
$$

We denote

$$
A_{O}^{(n+4)}=v, A_{O}^{(n+3)}=u, A_{O}^{(n+2)}=z, A_{O}^{(n+1)}=y, A_{O}^{(n)}=x
$$

and with formula (0.12), viz.

$$
A_{O}^{(n+5)}=A_{O}^{(n)}+5 D A_{0}^{(n+1)}+10 D^{2} A_{O}^{(n+2)}+10 D^{3} A_{0}^{(n+3)}+5 D^{4} A_{0}^{(n+4)} \text {. }
$$

We will also denote

$$
\begin{aligned}
& 5 D=a_{4}, 10 D^{2}=a_{3}, 10 D^{3}=a_{2}, 5 D^{4}=a_{1}, \\
& \left(a_{4}=b_{1}^{(0)} ; a_{3}=b_{2}^{(0)} ; a_{2}=b_{3}^{(0)}, a_{1}=b_{4}^{(0)}\right) .
\end{aligned}
$$

We then proceed as follows (in order to represent (6.2) as an expression in powers of $\mathrm{x}, \mathrm{y}, \mathrm{z}, \mathrm{u}, \mathrm{v})$ :

i) from the first row we subtract the $a_{1}$ multiple of the second row, then the $a_{2}$ multiple of the third row, then the $a_{3}$ multiple of the fourth row, then the $a_{4}$ multiple of the fifth row.

ii) from the second row we subtract the $a_{1}$ multiple of the third row, then the $a_{2}$ multiple of the fourth row, then the $a_{3}$ multiple of the fifth row.

iii) from the third row we subtract the $a_{1}$ multiple of the fourth row, then the $a_{2}$ multiple of the fifth row.

iv) from the fourth row we subtract the $a_{1}$ multiple of the fifth row, 
and obtain, always applying formula (0.12) and the notations (6.3), (6.4);

$\left|\begin{array}{ccccc}v-a_{1} u-a_{2} z-a_{3} y-a_{4} x & x & y & z & u \\ u-a_{1} z-a_{2} y-a_{3} x & v-a_{1} u-a_{2} z-a_{3} y & x+a_{4} y & y+a_{4} z & z+a_{4} u \\ z-a_{1} y-a_{2} x & u-a_{1} z-a_{2} y & v-a_{1} u-z_{2} z & x-a_{4} y+a_{3} z & y-a_{4} z+a_{3} u \\ y-a_{1} x & z-a_{1} y & u-a_{1} z & v-a_{1} u & x+a_{4} y+a_{3} z+a_{2} u \\ x & y & z & u & v\end{array}\right|=1(6 \cdot 5)$

with the values of $a_{1}, a_{2}, a_{3}, a_{4}$ from $(6.4), x, y, z, u, v$ from (6.3) where $n=0,1, \ldots$. The expansion of the determinant (6.5) would yield the expression. Even with $D=1$, it will still be very complicated. For $\mathrm{n}=0, \mathrm{x}=1, \mathrm{y}=\mathrm{z}=\mathrm{u}=\mathrm{v}=0$, the determinant in (6.5) becomes

$$
\left|\begin{array}{lllll}
-a_{4} & 1 & 0 & 0 & 0 \\
-a_{3} & 0 & 1 & 0 & 0 \\
-a_{2} & 0 & 0 & 1 & 0 \\
-a_{1} & 0 & 0 & 0 & 1 \\
1 & 0 & 0 & 0 & 0
\end{array}\right|=1
$$

and for $\mathrm{n}=\mathrm{I}, \mathrm{u}=\mathrm{z}=\mathrm{y}=\mathrm{x}=0, \mathrm{v}=\mathrm{I}$, the determinant becomes

$$
\left|\begin{array}{lllll}
1 & 0 & 0 & 0 & 0 \\
0 & 1 & 0 & 0 & 0 \\
0 & 0 & 1 & 0 & 0 \\
0 & 0 & 0 & 1 & 0 \\
0 & 0 & 0 & 0 & 1
\end{array}\right|=1
$$

but these elementary determinants can hardly serve as a verification for formula (6.5). For $\mathrm{n}=2$ the test is also simple.

Let try for $n=3$, $\left(A_{0}^{(3)}, A_{0}^{(4)}, A_{0}^{(5)}, A_{0}^{(6)}, A_{0}^{(7)}\right)=\left(0,0,1, a_{1}, a_{2}+a_{1}^{2}\right)=(x, y, z, u, v):$

$$
\left|\begin{array}{ccccc}
a_{2}+a_{1}^{2}-a_{1}^{2}-a_{2} & 0 & 0 & 1 & a_{1} \\
a_{1}-a_{1} & a_{2}+a_{1}^{2}-a_{1}^{2}-a_{2} & 0 & a_{4} & 1+a_{1} a_{4} \\
1 & a_{1}-a_{1} & a_{2}+a_{1}^{2}-a_{1}^{2}-a_{2} & a_{3} & a_{4}+a_{1} a_{3} \\
0 & 1 & a_{1}-a_{1} & a_{2}+a_{1}^{2}-a_{1}^{2} & a_{3}+a_{1} a_{2} \\
0 & 0 & 1 & a_{1} & a_{2}+a_{1}^{2}
\end{array}\right|=
$$




$$
=\left|\begin{array}{ccccc}
0 & 0 & 0 & 1 & a_{1} \\
0 & 0 & 0 & a_{4} & 1+a_{1} a_{4} \\
1 & 0 & 0 & a_{3} & a_{4}+a_{1} a_{3} \\
0 & 1 & 0 & a_{2} & a_{3}+a_{1} a_{2} \\
0 & 0 & 1 & a_{1} & a_{2}+a_{1}^{2}
\end{array}\right|=
$$

and subtracting the $a_{1}$ multiple of the fourth column from the fifth

$$
\begin{aligned}
& =\left|\begin{array}{lllll}
0 & 0 & 0 & 1 & 0 \\
0 & 0 & 0 & a_{4} & 1 \\
1 & 0 & 0 & a_{3} & a_{4} \\
0 & 1 & 0 & a_{2} & a_{3} \\
0 & 0 & 1 & a_{1} & a_{2}
\end{array}\right|= \\
& 1 \cdot\left|\begin{array}{llll}
0 & 0 & 1 & 0 \\
0 & 0 & a_{4} & 1 \\
1 & 0 & a_{2} & a_{3} \\
0 & 1 & a_{1} & a_{2}
\end{array}\right|=1 \cdot\left|\begin{array}{lll}
0 & 0 & 1 \\
1 & 0 & a_{3} \\
0 & 1 & a_{2}
\end{array}\right|=1 .
\end{aligned}
$$

7. FIFTH DEGREE IDENTITIES

As we have seen, the ACF of the fixed vector

$a^{(0)}=\left(w+4 D, w^{2}+3 D w+6 D^{2}, w^{3}+2 D w^{2}+3 D^{2} w+4 D^{3}, w^{4}+D w^{3}+D^{2} w^{2}+D^{3} w+D^{4}\right)$

is purely periodic with length of the primitive period $\ell=1$. Hence we have the formula

$$
\begin{aligned}
e^{n}= & \left(w^{4}+D w^{3}+D^{2} w^{2}+D^{3} w+D^{4}\right)^{n}= \\
& A_{0}^{(n)}+(w+4 D) A_{0}^{(n+1)}+\left(w^{2}+3 D w+6 D^{2}\right) A_{0}^{(n+2)}+ \\
& +\left(w^{3}+2 D w^{2}+3 D^{2} w+4 D^{3}\right) A_{0}^{(n+3)}+\left(w^{4}+D w^{3}+D^{2} w^{2}+D^{3} w+D^{4}\right) A_{O}^{(n+4)}, \\
& n=0,1,2, \ldots \\
& A_{0}^{(v)}(v=5,6, \ldots) \text { from }[1] .
\end{aligned}
$$

From (7.1) we obtain

$$
\begin{aligned}
\left(w^{4}+\right. & \left.D w^{3}+D^{2} w^{2}+D^{3} w+D^{4}\right)^{5 n}= \\
= & A_{0}^{(5 n)}+4 D A_{O}^{(5 n+1)}+6 D^{2} A_{O}^{(5 n+2)}+4 D^{3} A_{O}^{(5 n+3)}+D^{4} A_{O}^{(5 n+4)+}+ \\
& +\left(A_{0}^{(5 n+1)}+3 D A_{0}^{(5 n+2)}+3 D^{2} A_{O}^{(5 n+3)}+D^{3} A_{O}^{(5 n+4)}\right) w+ \\
& +\left(A_{O}^{(5 n+2)}+2 D A_{O}^{(5 n+3)}+D^{2} A_{O}^{(5 n+4)}\right) w^{2}+ \\
& +\left(A_{O}^{(5 n+3)}+D A_{O}^{(5 n+4)}\right) w^{3}+A_{O}^{(5 n+4)} w^{4} .
\end{aligned}
$$

We shall now arrange $\left(w^{4}+D w^{3}+D^{2} w^{2}+D^{3} w+D^{4}\right)^{5 n}$ in descending powers of $w$. The first step will be to achieve this arrangement in powers of $w^{5 s}$, 
$\mathrm{s}=0,1,2,3, \ldots, 4 \mathrm{n}$, since the highest power of $\mathrm{w}$ in that expression is $w^{20 n}$, so we look for the rational part of it. We have by the multinomial theorem

$$
\left.\begin{array}{l}
\left(w^{4}+D w^{3}+D^{2} w^{2}+D^{3} w+D^{4}\right)^{5 n}= \\
\sum_{4 y_{1}+3 y_{2}+2 y_{3}+y_{4}=k}\left(\begin{array}{l}
y_{1}+y_{2}+y_{3}+y_{4}+y_{5} \\
y_{1}, y_{2}, y_{3}, y_{4}, y_{5}
\end{array}\right){ }_{w}^{4 y_{1}+3 y_{2}+2 y_{3}+y_{4} \ldots D_{2}+2 y_{3}+3 y_{4}+4 y_{5}} \\
y_{2}+2 y_{3}+3 y_{4}+4 y_{5}=20 n-k, k=0,1, \ldots, 20 n
\end{array}\right\}
$$

since the sum of the exponents of $\mathrm{w}$ and $\mathrm{D}$ in the above expansion equals $20 n=k+(20 n-k)$. We also have from $(7 \cdot 3)$

$$
\begin{aligned}
& 4 y_{1}+4 y_{2}+4 y_{3}+4 y_{4}+4 y_{5}=20 n \\
& y_{1}+y_{2}+y_{3}+y_{4}+y_{5}=5 n .
\end{aligned}
$$

Since we are looking for 5-multiples of the exponents of $\mathrm{w}$ - hence also of D - we obtain from (7.3), (7.4):

The rational part in the expansion of $\left(w^{4}+D w^{3}+D^{2} w^{2}+D^{3} w+D^{4}\right)^{5 n}$ equals

$$
\begin{gathered}
\sum_{\sum_{i=1}^{4}}\left(\begin{array}{c}
5 n \\
y_{1}, y_{2}, y_{3}, y_{4}, y_{5}
\end{array}\right) m^{s} D^{20 n-5 s} \\
s \geq 0, m=w_{i}=5 s \leq 20 n \\
s \geq\left(D^{5}+1\right) .
\end{gathered}
$$

The equation $y_{2}+2 y_{3}+3 y_{4}+4 y_{5}=20 n-5 s$ follows from $y_{1}+y_{2}+y_{3}+y_{4}+y_{5}=5 n$ in the multinomial coefficient.

As an illustration to (7.5) we shall find the rational part in the expansion of $\left(w^{4}+D w^{3}+D^{2} w^{2}+D^{3} w+D^{4}\right)^{5}, n=1$. We obtain from (7.5) that this equals

$$
\begin{aligned}
& \sum_{i=1}^{4}\left(y_{1}, y_{2}, y_{3}, y_{4}, y_{5}\right) m^{s} D^{20-5 s} \\
& \sum_{i}(5-i) y_{i}=5 s \leq 20
\end{aligned}
$$

We solve the equations, $s=0,1,2,3,4$

$$
\begin{array}{cl}
\mathrm{s}=0 ; & 4 \mathrm{y}_{1}+3 \mathrm{y}_{2}+2 \mathrm{y}_{3}+\mathrm{y}_{4}=0, \mathrm{y}_{1}+\mathrm{y}_{2}+\mathrm{y}_{3}+\mathrm{y}_{4}+\mathrm{y}_{5}=5 \\
\mathrm{y}_{1}=\mathrm{y}_{2}=\mathrm{y}_{3}=\mathrm{y}_{4}=0, \mathrm{y}=5 .
\end{array}
$$

The corresponding member in (7.6) equals

$$
\left(\begin{array}{l}
5 \\
5
\end{array}\right) \mathrm{m}^{0} \mathrm{D}^{20-0}=\mathrm{D}^{20} \text {. }
$$




$$
\begin{array}{ll}
\mathrm{s}=1 ; & 4 \mathrm{y}_{1}+3 \mathrm{y}_{2}+2 \mathrm{y}_{3}+\mathrm{y}_{4}=5, \mathrm{y}_{1}+\mathrm{y}_{2}+\mathrm{y}_{3}+\mathrm{y}_{4}+\mathrm{y}_{5}=5 \\
\mathrm{y}_{1}=\mathrm{y}_{4}=1 ; \mathrm{y}_{2}=\mathrm{y}_{3}=0 ; \mathrm{y}_{5}=3 . \\
\mathrm{y}_{1}=0 ; \mathrm{y}_{2}=\mathrm{y}_{3}=1 ; \mathrm{y}_{4}=0 ; \mathrm{y}_{5}=3 \\
\mathrm{y}_{1}=0 ; \mathrm{y}_{2}=1 ; \mathrm{y}_{3}=0 ; \mathrm{y}_{4}=2 ; \mathrm{y}_{5}=2 \\
\mathrm{y}_{1}=\mathrm{y}_{2}=0 ; \mathrm{y}_{3}=1 ; \mathrm{y}_{4}=3 ; \mathrm{y}_{5}=1 \\
\mathrm{y}_{1}=\mathrm{y}_{2}=0 ; \mathrm{y}_{3}=2 ; \mathrm{y}_{4}=1 ; \mathrm{y}_{5}=2 \\
\mathrm{y}_{1}=\mathrm{y}_{2}=\mathrm{y}_{3}=\mathrm{y}_{5}=0 ; \mathrm{y}_{4}=5 .
\end{array}
$$

The corresponding member in (7.6) equals

$$
\begin{aligned}
& {\left[\left(\begin{array}{c}
5 \\
1,1,0,0,3
\end{array}\right)+\left(\begin{array}{c}
5 \\
0,1,1,0,3
\end{array}\right)+\left(\begin{array}{c}
5 \\
0,1,0,2,2
\end{array}\right)+\left(\begin{array}{c}
5 \\
0,0,1,3,1
\end{array}\right)+\right.} \\
& \left.\left(\begin{array}{c}
5 \\
0,0,2,1,2
\end{array}\right)+\left(\begin{array}{c}
5 \\
0,0,0,0,5
\end{array}\right)\right] \mathrm{mD}^{15}=121 \mathrm{mD}^{15} . \\
& \mathrm{s}=2 ; 4 \mathrm{y}_{1}+3 \mathrm{y}_{2}+2 \mathrm{y}_{3}+\mathrm{y}_{4}=10 ; \mathrm{y}_{1}+\mathrm{y}_{2}+\mathrm{y}_{3}+\mathrm{y}_{4}+\mathrm{y}_{5}=5 .
\end{aligned}
$$

We shall write $\left(\mathrm{y}_{1}, \mathrm{y}_{2}, \mathrm{y}_{3}, \mathrm{y}_{4}, \mathrm{y}_{5}\right)$ for the solution of the above linear equations.

$$
\begin{array}{lll}
(2,0,1,0,2) ; & (2,0,0,2,1) ; & (1,2,0,0,2) ; \\
(1,1,1,1,1) ; & (0,3,0,1,1) ; & (0,2,2,0,1) ; \\
(0,1,3,1,0) ; & (0,2,1,2,0) ; & (0,1,3,1,0) ; \\
(0,0,5,0,0) ; & (1,0,3,0,1) ; & (1,0,2,2,0) .
\end{array}
$$

The corresponding member in (7.6) equals

$$
\begin{aligned}
& {\left[(2,0,1,0,2)+\left(\begin{array}{c}
5 \\
2,0,0,2,1
\end{array}\right)+\left(\begin{array}{c}
5 \\
1,2,0,0,2
\end{array}\right)+\left(\begin{array}{c}
5 \\
1,1,1,1,1
\end{array}\right)+\right.}
\end{aligned}
$$

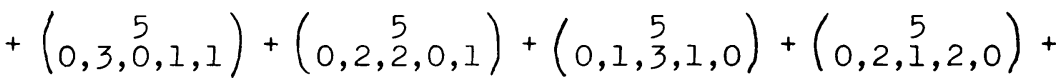

$$
\begin{aligned}
& \left.+\left(\begin{array}{l}
5 \\
0,1,3,1,0
\end{array}\right)+(0,0,5,0,0)+(1,0,3,0,1)+\left(\begin{array}{c}
5 \\
1,0,2,2,0
\end{array}\right)\right]= \\
& =(30+30+30+120+20+30+20+30+20+1+20+30) \mathrm{m}^{2} \mathrm{D}^{10}= \\
& =\underline{381 \mathrm{~m}^{2} \mathrm{D}^{10}} \text {. } \\
& \mathrm{s}=3 ; 4 \mathrm{y}_{1}+3 \mathrm{y}_{2}+2 \mathrm{y}_{3}+\mathrm{y}_{4}=15 ; \mathrm{y}_{1}+\mathrm{y}_{2}+\mathrm{y}_{3}+\mathrm{y}_{4}+\mathrm{y}_{5}=5 \text {. } \\
& (3,1,0,0,1) ; \quad(2,2,0,1,0) ; \quad(3,0,1,1,0) ; \\
& (2,1,2,0,0) ; \quad(1,3,1,0,0) ; \quad(0,5,0,0,0) \text {. }
\end{aligned}
$$

The corresponding members in $(7.6)$ equals

$$
\begin{aligned}
& {\left[\left(\begin{array}{c}
5 \\
3,1,0,0,1
\end{array}\right)+\left(\begin{array}{c}
5 \\
2,2,0,1,0
\end{array}\right)+\left(\begin{array}{c}
5 \\
3,0,1,1,0
\end{array}\right)+(2,1,2,0,0)+\right.} \\
& \left.+\left(\begin{array}{c}
5 \\
+3,1,0,0
\end{array}\right)+\left(\begin{array}{c}
5 \\
0,5,0,0,0
\end{array}\right)\right] \mathrm{m}^{3} \mathrm{D}^{5}= \\
& =(20+30+20+30+20+1) \mathrm{m}^{3} \mathrm{D}^{5}=121 \mathrm{~m}^{3} \mathrm{D}^{5} .
\end{aligned}
$$


$\mathrm{s}=4 ; 4 \mathrm{y}_{1}+3 \mathrm{y}_{2}+2 \mathrm{y}_{3}+\mathrm{y}_{4}=20 ; \mathrm{y}_{1}+\mathrm{y}_{2}+\mathrm{y}_{3}+\mathrm{y}_{4}+\mathrm{y}_{5}=5$.

The only solution is $(5,0,0,0,0)$ and the corresponding member in $(7.6)$ equals

$$
(5,0,0,0,0) m^{4}=m^{4} \text {. }
$$

Thus the formula (7.5) yields, for $n=1$, the sum

$$
m^{4}+121 m^{3} D^{5}+381 m^{2} D^{10}+121 m D^{15}+D^{20} \text {. }
$$

From the other side we have

$$
\begin{aligned}
\left(w^{4}+D w^{3}+D^{2} w^{2}+D^{3} w+D^{4}\right)^{5}= \\
=w^{20}+5 w^{19} D+15 w^{18} D^{2}+35 w^{17} D^{3}+70 w^{16} D^{4}+ \\
+121 w^{15} D^{5}+185 w^{14} D^{6}+255 w^{13} D^{7}+320 w^{12} D^{8}+ \\
+365 w^{11} D^{9}+381 D^{10} w^{10}+365 w^{9} D^{11}+320 w^{8} D^{12}+ \\
+255 w^{7} D^{13}+185 w^{6} D^{14}+121 w^{5} D^{15}+70 w^{4} D^{16}+ \\
+35 w^{3} D^{17}+15 w^{2} D^{18}+5 w D^{19}+D^{20} .
\end{aligned}
$$

That the expansion in (7.8) is symmetric (the coefficients of $w^{i} D^{20-i}$ and $w^{20-i} D^{i}, i=0,1, \ldots, 20$, are equal) is clear. The rational part equals

$$
\begin{aligned}
w^{20} & +121 w^{15} D^{5}+381 w^{10} D^{10}+121 w^{5} D^{15}+D^{20}= \\
& =m^{4}+121 m^{3} D^{5}+381 m^{2} D^{2}+121 m D^{15}+D^{20}
\end{aligned}
$$

as should be by (7.7).

Comparing formulas (7.2) with (7.5), we obtain the identity

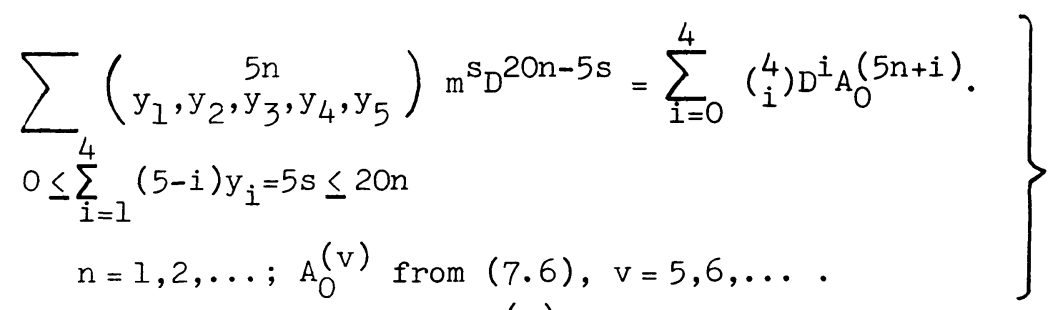

Substitution of the values of $A_{O}^{(v)}$ from (7.6) in (7.9) would yield a new expression for (7.9). The reader can prove the statements:

The coefficients of $w$ in the expansion of $\left(w^{4}+D w^{3}+D^{2} w^{2}+D^{3} w+D^{4}\right)^{5 n}$ equals

$$
\begin{gathered}
\sum_{i=1}^{4}\left(\begin{array}{c}
y_{1}, y_{2}, y_{3}, y_{4}, y_{5} \\
\sum_{i}
\end{array}\right) m^{s} D^{20 n-5 s-1} y_{i}=5 s+1 \leq 20 n ; \\
s=0,1, \ldots, 4 n-1 .
\end{gathered}
$$

Furthermore, the coefficients of $w^{i}$ in the expansion of $\left(w^{4}+D w^{3}+D^{2} w^{2}+D^{3} w+D^{4}\right)^{5 n}$ equal, with $i=0,1,2,3,4$, 


$$
\begin{aligned}
& \sum{ }_{i \leq}\left(y_{1}, y_{2}, y_{3}, y_{4}, y_{5}\right) m^{s} D^{20 n-5 s-i} \\
& \quad(5-i) y_{i}=5 s+i \leq 20 n \\
& s=0,1, \ldots, 4 n-1, i=0,1, \ldots, 4 .
\end{aligned}
$$

Comparing (7.2) with (7.11) we have finally the five identities,

$$
\begin{gathered}
\left.\sum_{i \leq \sum_{j=0}^{4}(5-j) y_{j}=5 s+i \leq 20 n} \begin{array}{c}
5 n \\
y_{1}, y_{2}, y_{3}, y_{4}, y_{5}
\end{array}\right) m^{s} D^{20 n-5 s-i}= \\
=\sum_{j=0}^{4-i}\left(\begin{array}{c}
4-i \\
j
\end{array}\right) D^{j} A_{0}(5 n+i+j) \\
i=0,1,2,3,4 .
\end{gathered}
$$

We shall give a verification for formula (7.12) with $i=0$, formula (7.9), $\mathrm{D}=1, \mathrm{n}=1$; we have $m=\mathrm{D}^{3}+1=2, \mathrm{~A}_{0}^{(0)}=1, \mathrm{~A}_{0}^{(1)}=\mathrm{A}_{0}^{(2)}=\mathrm{A}_{0}^{(3)}=\mathrm{A}_{0}^{(4)}=$ $0, A_{O}^{(n+5)}=A_{O}^{(n)}+5 A_{0}^{(n+1)}+10 A_{O}^{(n+2)}+10 A_{O}^{(n+3)}+5 A_{0}^{(n+4)}, A_{0}^{(5)}=1, A_{0}^{(6)}=5$, $A_{O}^{(7)}=35, A_{O}^{(8)}=235, A_{O}^{(9)}=1580$.

This yields

$$
16+121 \cdot 8+381 \cdot 4+121 \cdot 2+1=1+20+210+940+1580=2751 .
$$

It is also easy to verify the identities (7.12) for $\mathrm{n}=2$.

8. MORE ABOUT UNITS AND IDENTITIES

$$
\begin{aligned}
& \text { Since } w^{5}-D^{5}=(w-D)\left(w^{4}+D w^{3}+D^{2} w^{2}+D^{3} w+D^{4}\right)=1 \text {, we have also, } \\
& \left(w^{5}=D^{5}+1\right) \\
& e^{-v}=(w-D)^{v}=\frac{1}{\left(w^{4}+D w^{3}+D^{2} w^{2}+D^{3} w+D^{4}\right)^{4}} \text {, }
\end{aligned}
$$

and with formula (7.2), and setting $v=5 n$, 


$$
\left.\begin{array}{l}
(w-D)^{5 n}=\frac{1}{a_{5}+a_{4} w+a_{3} w^{2}+a_{2} w^{3}+a_{1} w^{4}} \\
a_{5-i}=\sum_{j=0}^{4-i}\left(\begin{array}{c}
4-i \\
j
\end{array}\right) D^{j} A_{0}^{(5 n+i+j)}, i=0, \ldots, 4 \text { (from (7.12)). }
\end{array}\right\}
$$

We shall now rationalize the denominator in (8.1) and demand

$$
I=\left(a_{5}+a_{4} w+a_{3} w^{2}+a_{2} w^{3}+a_{1} w^{4}\right)\left(c_{1}+c_{2} w+c_{3} w^{2}+c_{4} w^{3}+c_{5} w^{5}\right) \text {. }
$$

Expanding (8.2), with $m=w^{5}=D^{5}+1$, we obtain

$$
\begin{aligned}
& a_{5} c_{1}+m a_{1} c_{2}+m a_{2} c_{3}+m a_{3} c_{4}+m a_{4} c_{5}=1 \\
& a_{4} c_{1}+a_{5} c_{2}+m a_{1} c_{3}+m a_{2} c_{4}+m a_{3} c_{5}=0 \\
& a_{3} c_{1}+a_{4} c_{2}+a_{5} c_{3}+m a_{1} c_{4}+m a_{2} c_{5}=0 \\
& a_{2} c_{1}+a_{3} c_{2}+a_{4} c_{3}+a_{5} c_{4}+m a_{1} c_{5}=0 \\
& a_{1} c_{1}+a_{2} c_{2}+a_{3} c_{3}+a_{4} c_{4}+a_{5} c_{5}=0 .
\end{aligned}
$$

The determinant of the system of linear equations (8.3) equals, interchanging columns with rows,

$$
\Delta=\left|\begin{array}{ccccc}
a_{5} & a_{4} & a_{3} & a_{2} & a_{1} \\
m a_{1} & a_{5} & a_{4} & a_{3} & a_{2} \\
m a_{2} & m a_{1} & a_{5} & a_{4} & a_{3} \\
m a_{3} & m a_{2} & m a_{1} & a_{5} & a_{4} \\
m a_{4} & m a_{3} & m a_{2} & m a_{1} & a_{5}
\end{array}\right| .
$$

Now, the reader will verify that the field equation of $e^{5 n}=a_{5}+a_{4} w+a_{3} w^{2}+a_{2} w^{3}+a_{1} w^{4}$ has exactly the free element $=1$, since $e$ is a unit, as in case $n=3$. We thus obtain

$$
(w-D)^{5 n}=c_{1}+c_{2} w+c_{3} w^{2}+c_{4} w^{3}+c_{5} w^{4} \text {. }
$$

Expanding $(w-D)^{5 n}$ we obtain the result. The rational part in the expansion of $(8.5)$ equals

$$
\sum_{i=0}^{n}(-1)^{i} D^{5 i} w^{5 n-5 i} \text {. }
$$

Comparing (8.6) with $c_{1}$ and calculating $c_{1}$ from (8.3), (8.4), we obtain the identity, with $\mathrm{w}^{5}=\mathrm{m}=\mathrm{D}^{5}+1$ 


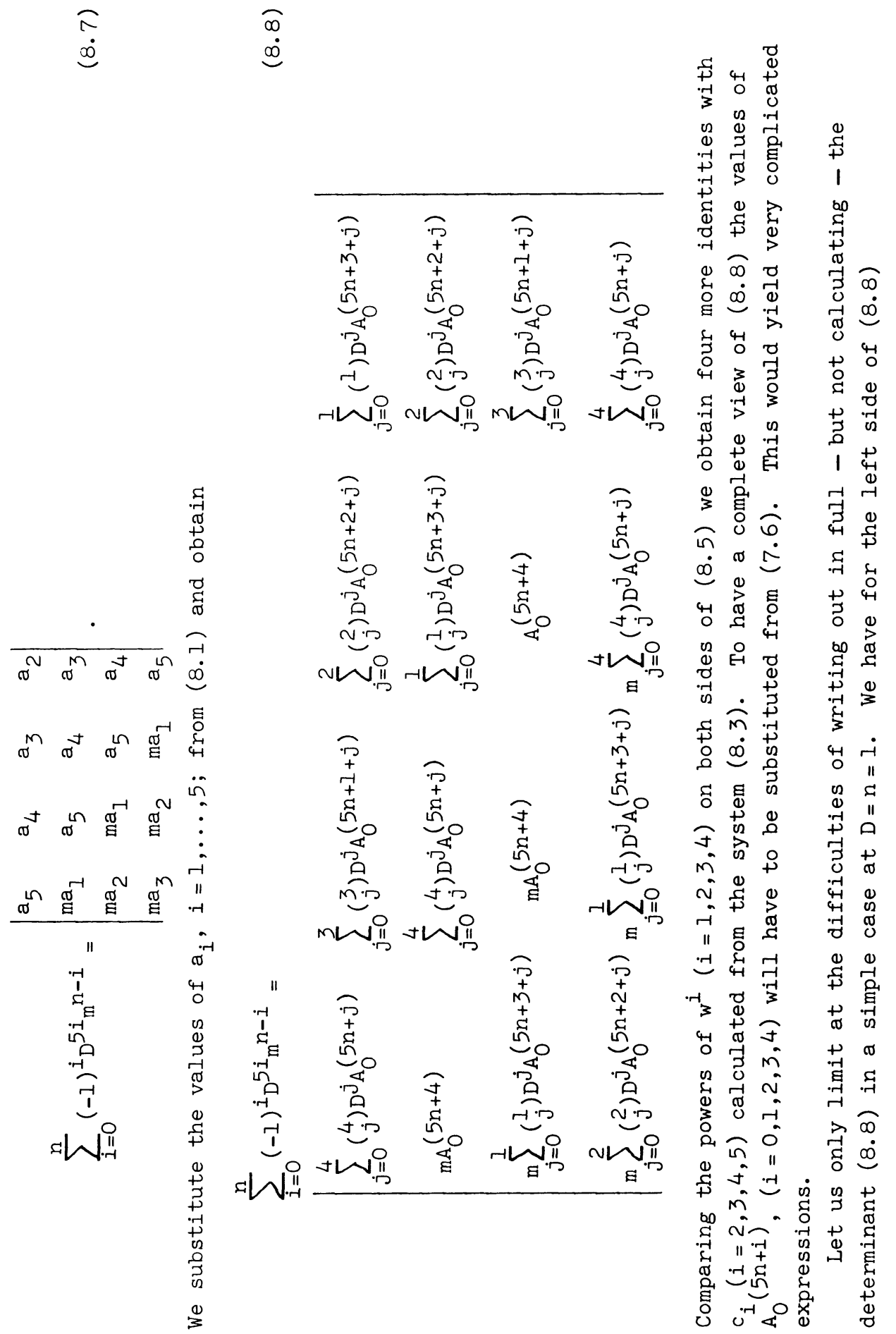




$$
\sum_{i=0}^{1}(-1)^{i} D^{5 i} m^{n-i}=m-D^{5}=1 .
$$

For the right side we calculate

$$
A_{O}^{(5)}=1, A_{O}^{(6)}=5, A_{O}^{(7)}=35, A_{O}^{(8)}=235, A_{O}^{(9)}=1580 .
$$

Thus the determinant (8.8) becomes, with the values from (8.1), viz.

$$
\begin{aligned}
& a_{5}=\sum_{j=0}^{4}\left(\begin{array}{l}
4 \\
j
\end{array}\right) A_{0}^{(5+j)}=1+4 \cdot 5+6 \cdot 35+4 \cdot 235+1580=2751 \text {, } \\
& a_{4}=\sum_{j=0}^{3}\left(\begin{array}{l}
3 \\
j
\end{array}\right) A_{0}^{(6+j)}=5+3 \cdot 35+3 \cdot 235+1580=2395 \text {, } \\
& a_{3}=\sum_{j=0}^{2}\left(\begin{array}{l}
2 \\
j
\end{array}\right) A_{0}^{(7+j)}=35+2 \cdot 235+1580=2085 \text {, } \\
& a_{2}=\sum_{j=0}^{I}\left(\begin{array}{l}
1 \\
j
\end{array}\right) A_{0}^{(8+j)}=235+1580=1815 \text {, } \\
& a_{1}=\sum_{j=0}^{0}\left(\begin{array}{l}
0 \\
j
\end{array}\right) A_{0}^{(9+j)}=1580, \quad m=2 \text {, } \\
& \left|\begin{array}{llll}
2751 & 2395 & 2085 & 1815 \\
3160 & 2751 & 2395 & 2085 \\
3630 & 3160 & 2751 & 2395 \\
4170 & 3630 & 3160 & 2751
\end{array}\right|=1 .
\end{aligned}
$$

Thus formula (8.8) has been verified for $D=n=1$. The entries in the right hand determinant become a challenge for $n, D>1$.

On the combined subject of this paper about "Diophantine Equations, Units and Identities" there is not much literature, but I cannot finish without naming the literature in each of the three above mentioned subjects without indicating at the very end, some papers which have been most useful in my paper.

\section{REFERENCES}

1. BAICA, M., An algorithm in a complex field and its application to the calculation of units, Pacific J. Math., Vol. 110, No. I, (1984), 21-40.

2. BAICA, M. , Approximation of irrationals, Internat. J. Math\& Math. Sci. , Vol. 8, No. 2 (1985), 303-320.

3. BERGMANN, G., Beispiele numerischer Einheitenbestimmung, Math. Ann. 1671 (1966) 143-168. MR 35 No. 158.

4. BELLEVIC, K. K., On units in algebraic fields of third and fourth degree, Mat. Sb. 40 (82), (1956), 123-136 (Russian) MR 19.533. 
5. BERNSTEIN, L., The Jacobi-Perron algorithm, its theory and applications. Springer, Berlin-Heidelberg-New York. Lect. Notes. Math. 207 (1971).

6. CARLITZ, L., Some combinatorial identities of Bernstein, SIAM J. Math. Analysis 9 (1978), 65-75.

7. CARLITZ, L., Recurrences of the third order and related combinatorial identities, Fibonacci Quarterly 16 (1978), 11-18.

8. DELONE, B. N. and FADDEEV, D. K., The theory of Irrationalities of the third degree, Translation of Math. Monographs, Amer. Math. Soc. , 10 (1964), 82-136, 316-449.

9. MAHLER, K., Periodic algorithms for algebraic number fields, Lectures given at the Fourth Summer Research Institute at the Australian Mathematical Soc., held at the University of Sydney, January, 1964.

10. ZASSENHAUS, H. On the units of orders, J. of Algebra 20, (1972), 368-395. 


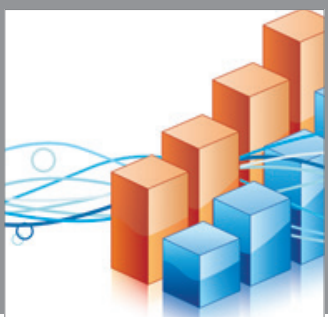

Advances in

Operations Research

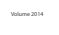

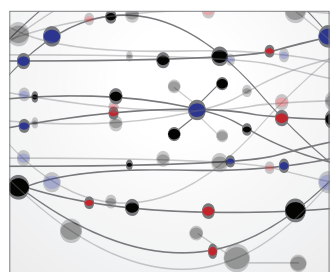

\section{The Scientific} World Journal
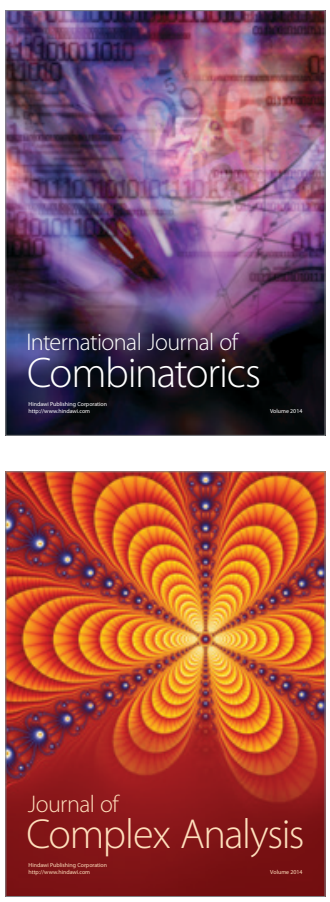

International Journal of

Mathematics and

Mathematical

Sciences
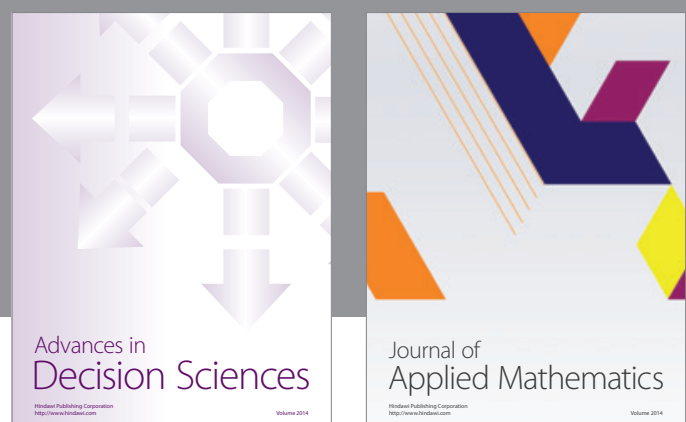

Journal of

Applied Mathematics
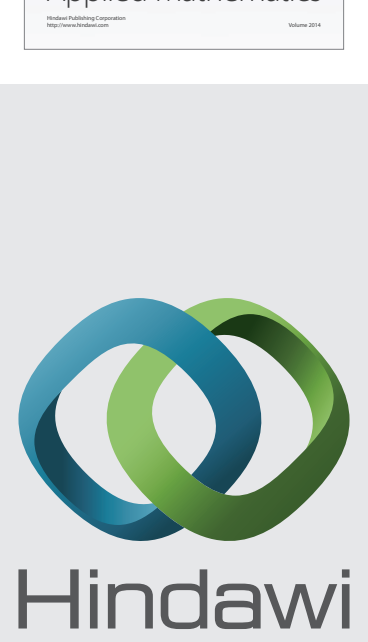

Submit your manuscripts at http://www.hindawi.com
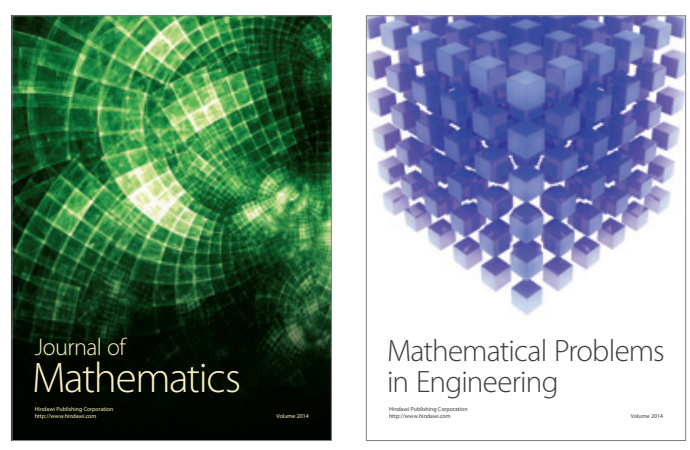

Mathematical Problems in Engineering
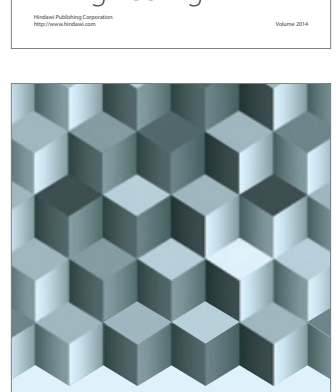

Journal of

Function Spaces
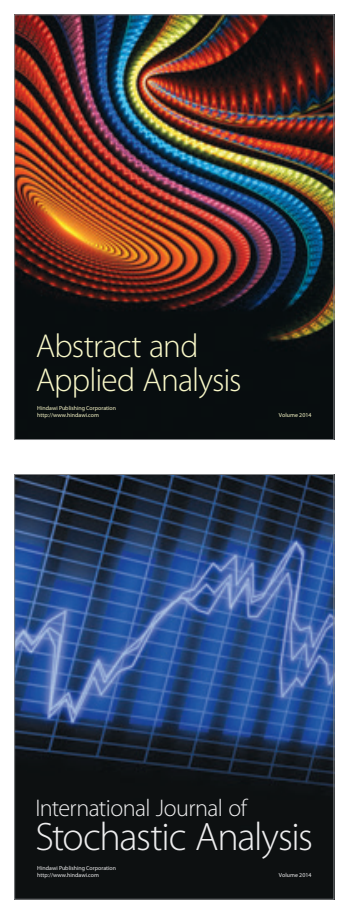

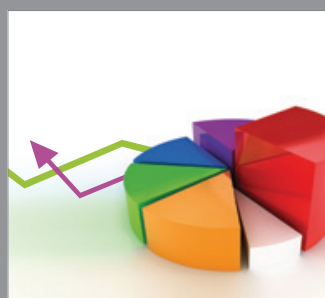

ournal of

Probability and Statistics

Promensencen
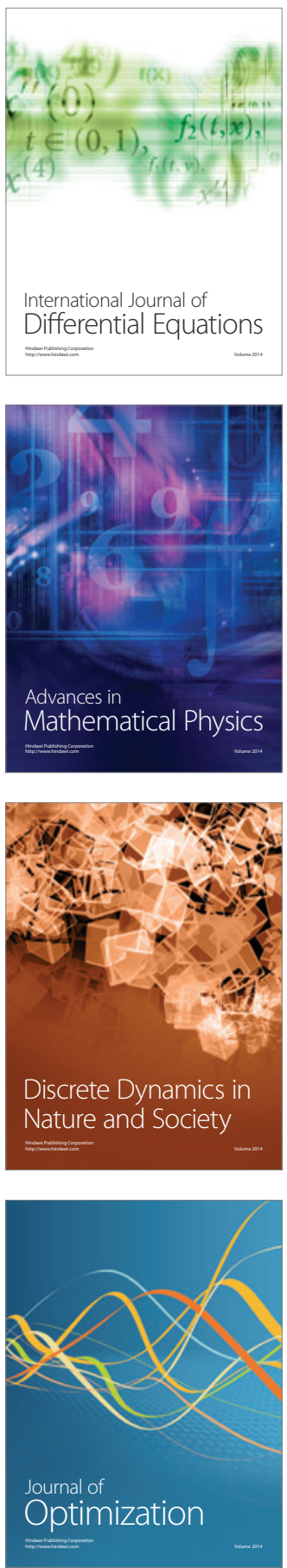